\title{
EFSUMB President Paul S Sidhu writes
}

We look forward to the New Year and 2018 promises to be a busy year for EFSUMB.

During the EUROSON meeting in Ljubljana, Slovenia the Board of Delegates met, with the composition of the various committees of EFSUMB seeing new blood which by now is firmly in place and engaging in the new tasks ahead. A number of long-standing committee members have stepped down, including Gail ter Haar (Safety), Christoph F Dietrich (Executive Board) and Michael Bachman-Nielsen (Publications) amongst others, all having devoted considerable time to the progress of EFSUMB. The President thanks all the previous committee members for their tireless efforts over the years. With new energy on the various committees we can look forward in 2018 to continuing innovation and policy making.
In the last year we have had much success under the auspices of the past President, Odd Helge Gilja, notably in the publication of two sets of EFSUMB guidelines (Liver elastography and Gastrointestinal) and one statement (Paediatric CEUS), all well received by the ultrasound community worldwide, with immediate impact as demonstrated by citations in the medical literature. In 2018, the update on non-liver CEUS will be published. In addition the steering committee and contributors will meet in February to put the final touches to a new set of guidelines related to non-liver elastography, likely to be completed and ready for publication in 2019. A further number of possible guidelines are under consideration and will be progressed over the coming year. None of these guidelines would be possible without the co-operation of all experts within the European ultrasound community who contribute their time and expertise so generously. We will continue to endeavour to publish the guidelines in our journal, European Journal of Ultrasound (Ultraschall in der Medizin), which continues to be a well-respected publication with a high impact factor. Needless to say in order to maintain the integrity of the journal all manuscripts undergo a rigid peer-review process, and this includes the guidelines produced by EFSUMB. The safety committee have been tasked by the journal editors to produce a series of continuing education articles (CME) on all aspects of ultrasound safety; expect to see these articles published regularly over the coming few years. 
The next EUROSON meeting will take place this year in Poznan, Poland form the 6-9 September in conjunction with the Polish Society. Preparations are well advanced with a lively program scheduled in the delightful setting of the charming city of Poznan. This year there is an ultra-early registration fee set at a very reasonable cost; look on the website and book now to take advantage of this extremely generous registration fee. We welcome as many as possible of the EFSUMB members to join us for the education and, of course, meeting old and new friends.

There will be a busy year of planned EFSUMB Schools in 2018, with courses on MSK, neonatal brain, chest and of course CEUS (London, Munich, Hong Kong and Berne) continuing the commitment of EFSUMB to continuing education. Please review these courses on the website and book early as the courses are very popular. Over the last year, EFSUMB has introduced webinars, so far dealing with aspects of CEUS. Another is planned for the $19^{\text {th }}$ January dealing with Intracavity CEUS, a niche topic but so very useful as a problem solving tool. Registration for these webinars is free and has proved to be popular. We are currently exploring the expansion of this method of education, taking topics from outside CEUS, and would value contributions and ideas from all members. We are actively exploring industry support for these webinars, in order to continue with an expanded program.

Going forward, we anticipate a very busy 2018, with all the committees working to produce many new documents, policies and guidelines. The website will continue to expand and include many new educational aspects, with continuing development of mobile information portals. I hope this year will be beneficial to all members of EFSUMB!

\section{Professor Paul S. Sidhu President of EFSUMB}

